TRANSACTIONS OF THE

AMERICAN MATHEMATICAL SOCIETY

Volume 352, Number 2, Pages 515-524

S 0002-9947(99)02253-9

Article electronically published on March 18, 1999

\title{
THE CALABI INVARIANT AND THE EULER CLASS
}

\author{
TAKASHI TSUBOI
}

\begin{abstract}
We show the following relationship between the Euler class for the group of the orientation preserving diffeomorphisms of the circle and the Calabi invariant for the group of area preserving diffeomorphisms of the disk which are the identity along the boundary. A diffeomorphism of the circle admits an extension which is an area preserving diffeomorphism of the disk. For a homomorphism $\psi$ from the fundamental group $\left\langle a_{1}, \cdots, a_{2 g} ;\left[a_{1}, a_{2}\right] \cdots\left[a_{2 g-1}, a_{2 g}\right]\right\rangle$ of a closed surface to the group of the diffeomorphisms of the circle, by taking the extensions $\widetilde{\psi\left(a_{i}\right.}$ ) for the generators $a_{i}$, one obtains the product $\left[\widetilde{\psi\left(a_{1}\right)}, \widetilde{\psi\left(a_{2}\right)}\right] \cdots\left[\widetilde{\psi\left(a_{2 g-1}\right), \widetilde{\psi\left(a_{2 g}\right.}}\right]$ of their commutators, and this is an area preserving diffeomorphism of the disk which is the identity along the boundary. Then the Calabi invariant of this area preserving diffeomorphism is a non-zero multiple of the Euler class of the associated circle bundle evaluated on the fundamental cycle of the surface.
\end{abstract}

\section{INTRODUCTION}

For a compact manifold $M$ with boundary, it is interesting to investigate the relationship between the group $\operatorname{Diff}(M)$ of the diffeomorphisms of the manifold and the group Diff $(\partial M)$ of the diffeomorphisms of its boundary.

To investigate $\operatorname{Diff}(\partial M)$ or its subgroups, it is reasonable to impose some restrictions on the diffeomorphisms of $M$. The important example in mind is that the conformal diffeomorphisms of $S^{q-1}=\partial D^{q}$ correspond bijectively to the conformal diffeomorphisms of $D^{q}$. This shows that the condition of having an extension which is a conformal diffeomorphism of $D^{q}$ is too restrictive. So we look at some milder conditions for the extension and in this paper we treat with the extensions which are volume preserving diffeomorphisms.

In fact, we look at the simplest case, namely, the relationship between the group Diff ${ }_{\Omega}\left(D^{2}\right)$ of the area preserving diffeomorphisms of the disk and the group $\operatorname{Diff}\left(S^{1}\right)_{0}$ of the orientation preserving diffeomorphisms of the circle.

Let $\operatorname{Diff}_{\Omega}\left(D^{2}\right)$ denote the group of the $C^{\infty}$ diffeomorphisms of the disk which preserve the volume form $\Omega$. Here we consider the usual volume form $\Omega=d x \wedge d y$ on the unit disk $D^{2}$. We show later that any orientation preserving diffeomorphism of the circle extends as an area preserving diffeomorphism of the disk.

Received by the editors June 6, 1997 and, in revised form, September 12, 1997.

1991 Mathematics Subject Classification. Primary 57R32, 53C15; Secondary 57R50, 58H10, $53 \mathrm{C} 12$.

Key words and phrases. Area preserving diffeomorphisms, classifying spaces, foliated circle bundles, foliated disk bundles, Calabi invariant, Euler class.

The author was supported in part by Grant-in-Aid for Scientific Research 07454013 and 09440028, Ministry of Education, Science, Sports and Culture, Japan.

(C)1999 American Mathematical Society 
Let $\Sigma_{\mathbf{g}}$ denote the closed oriented surface of genus $\mathbf{g}$. Its fundamental group has the well known presentation

$$
\left\langle a_{1}, \cdots, a_{2 \mathbf{g}} ;\left[a_{1}, a_{2}\right] \cdots\left[a_{2 \mathbf{g}-1}, a_{2 \mathbf{g}}\right]\right\rangle .
$$

Let $\psi: \pi_{1}\left(\Sigma_{\mathbf{g}}\right) \longrightarrow \operatorname{Diff}\left(S^{1}\right)_{0}$ be a homomorphism, where $\operatorname{Diff}\left(S^{1}\right)_{0}$ denotes the group of $C^{\infty}$ diffeomorphisms of the circle isotopic to the identity with the $C^{\infty}$ topology. Then we have the extensions $h_{i} \in \operatorname{Diff}_{\Omega}\left(D^{2}\right)$ of $g_{i}=\psi\left(a_{i}\right) \in \operatorname{Diff}\left(S^{1}\right)_{0}$ and the product $\left[h_{1}, h_{2}\right] \cdots\left[h_{2 \mathrm{~g}-1}, h_{2 \mathrm{~g}}\right]$ of commutators is an area preserving diffeomorphism of the disk which is the identity along the boundary.

Let $\operatorname{Diff}_{\Omega}\left(D^{2}, \partial D^{2}\right)$ denote the group of area preserving diffeomorphisms of the disk which are the identity along the boundary. There is a homomorphism $\rho: \operatorname{Diff}_{\Omega}\left(D^{2}, \partial D^{2}\right) \longrightarrow \mathbf{R}$ which is similar to that given by Calabi $([2])$; see $[1$, Proposition II.4.3].

We prove the following theorem.

Theorem A. Let $\psi: \pi_{1}\left(\Sigma_{\mathbf{g}}\right) \longrightarrow \operatorname{Diff}\left(S^{1}\right)_{0}$ be a homomorphism, where

$$
\pi_{1}\left(\Sigma_{\mathbf{g}}\right)=\left\langle a_{1}, \cdots, a_{2 \mathbf{g}} ;\left[a_{1}, a_{2}\right] \cdots\left[a_{2 \mathbf{g}-1}, a_{2 \mathbf{g}}\right]\right\rangle .
$$

Let $h_{i} \in \operatorname{Diff}_{\Omega}\left(D^{2}\right)$ be an extension of $g_{i}=\psi\left(a_{i}\right)$. Then $\rho\left(\left[h_{1}, h_{2}\right] \cdots\left[h_{2 \mathbf{g}-1}, h_{2 \mathbf{g}}\right]\right)$ is a non-zero multiple of the Euler class of the associated $S^{1}$ or $D^{2}$ bundle evaluated on the fundamental class $\left[\Sigma_{\mathbf{g}}\right]$.

As a corollary to this theorem, we see that a foliated 2-disk bundle over a surface whose global holonomy is in $\operatorname{Diff}_{\Omega}\left(D^{2}\right)$ is topologically trivial. This fact has been known by a much stronger result of Mitsumatsu ([11], [7]).

This paper is organized as follows. In $\S 1$, we give the definition of Calabi invariants. The Calabi invariants are usually considered for the group of symplectic diffeomorphisms with compact support of a symplectic manifold without boundary. In particular, in the 2-dimensional case they are considered for the group of area preserving diffeomorphisms with compact support of $\mathbf{R}^{2}$ or $\operatorname{Int} D^{2}$. For our purpose, it is necessary to define them for the group of area preserving diffeomorphisms of $D^{2}$. $\S 1$ consists of the verification of the formulae in [1] in our context. We define the Calabi invariant $\rho$ on $\operatorname{Diff}_{\Omega}\left(D^{2}, \partial D^{2}\right)$ and $R$ on $\widetilde{\operatorname{Diff}}_{\Omega}\left(D^{2}\right)$, the universal covering group of Diff $\Omega\left(D^{2}\right)$.

In $\S 2$, we show that for any compact manifold $M$, the homomorphism of the restriction from the group $\operatorname{Diff}_{\Omega}(M)$ of volume preserving diffeomorphisms of $M$ to the group $\operatorname{Diff}(\partial M)$ of diffeomorphisms of $\partial M$ is surjective on the identity component. This surjectivity has already been obtained by Krygin ([8]). Using Moser's homotopy ([12]), we also show that the inclusion map of the group $\operatorname{Diff}_{\Omega}(M, \partial M)$ of volume preserving diffeomorphisms of $M$ being the identity along $\partial M$ in the group $\operatorname{Diff}(M, \partial M)$ of diffeomorphisms of $M$ being the identity along $\partial M$ is a weak homotopy equivalence. Then a result of Smale ([14]) implies that $\operatorname{Diff}_{\Omega}\left(D^{2}, \partial D^{2}\right)$ is contractible. This allows us to compute the difference of $\rho$ and $R$.

In $\S 3$, we prove our theorem in the language of universal 2-cocycles on $\operatorname{Diff}\left(S^{1}\right)$. Then this implies Theorem A. We also include a discussion on the Euler class of the foliated disk bundles with transverse invariant volume form.

I would like to thank Marco Brunella for very helpful discussions on the Calabi invariant and the Euler class. I also thank l'Institut Fourier as well as the host, Vlad Sergiescu, for their warm hospitality during my stay in the spring of 1997, where I could finish writing this paper. 


\section{Calabi invariants}

Let $D^{2}$ denote the disk $\left\{(x, y) \in \mathbf{R} ; x^{2}+y^{2} \leq 1\right\}$ with the volume form $\Omega=$ $d x \wedge d y$. Let $\operatorname{Diff}_{\Omega}\left(D^{2}, \partial D^{2}\right)$ denote the group of area preserving diffeomorphisms of the disk which are the identity along the boundary. We are going to define the homomorphism $\rho: \operatorname{Diff}_{\Omega}\left(D^{2}, \partial D^{2}\right) \longrightarrow \mathbf{R}$ in the statement of Theorem A. (See $[1$, Proposition II.4.3] for the case of symplectic manifolds without boundary.)

Let $\lambda$ be a 1-form such that $d \lambda=\Omega$. For example, for the polar coordinate $(r, \theta)$ on $D^{2}$, put $\lambda=\left(r^{2} / 2\right) d \theta=(1 / 2)(x d y-y d x)$. For an element $h$ of $\operatorname{Diff}_{\Omega}\left(D^{2}, \partial D^{2}\right)$, we look at the 1 -form $h^{*} \lambda-\lambda$. Since $d \lambda=\Omega$, this is a closed form, we obtain a function $f(h, \lambda)$ such that $d f(h, \lambda)=h^{*} \lambda-\lambda$.

Let $Z=\partial / \partial \theta=x(\partial / \partial y)-y(\partial / \partial x)$ be the rotational vector field. Now on $\partial D$,

$$
\begin{aligned}
Z f(h, \lambda) & =i(Z) d f(h, \lambda)=i(Z)\left(h^{*} \lambda-\lambda\right) \\
& =i\left(h_{*} Z\right) \lambda-i(Z) \lambda=i(Z) \lambda-i(Z) \lambda=0 .
\end{aligned}
$$

Hence we choose $f(h, \lambda)$ which is zero along the boundary. We put

$$
\rho(h)=\int_{D^{2}} f(h, \lambda) \Omega .
$$

This integral does not depend on the choice of $\lambda$; see [4]. Note that for a function $u$ such that $\lambda^{\prime}-\lambda=d u$, one obtains

$$
f\left(h, \lambda^{\prime}\right)=f(h, \lambda)+(u \circ h-u)
$$

because $(u \circ h-u) \mid \partial D^{2}=0$ for $h \in \operatorname{Diff}_{\Omega}\left(D^{2}, \partial D^{2}\right)$.

It is easy to see that $\rho\left(h_{1} h_{2}\right)=\rho\left(h_{1}\right)+\rho\left(h_{2}\right)$. Note here that

$$
f\left(h_{1} h_{2}, \lambda\right)=h_{2}^{*} f\left(h_{1}, \lambda\right)+f\left(h_{2}, \lambda\right)
$$

because $f(h, \lambda)$ is zero along the boundary.

Thus we have shown the following lemma.

Lemma (1.1). $\rho: \operatorname{Diff}_{\Omega}\left(D^{2}, \partial D^{2}\right) \longrightarrow \mathbf{R}$ is a homomorphism.

Note that $\rho$ with respect to $\lambda=\left(r^{2} / 2\right) d \theta=(1 / 2)(x d y-y d x)$ can be defined for an area preserving diffeomorphism whose restriction to the boundary is a rotation.

Let Diff ${ }_{\Omega}\left(D^{2}\right)$ denote the group of the $C^{\infty}$ diffeomorphisms of the disk which preserve the volume form $\Omega$. This group is connected with respect to the $C^{\infty}$ topology as we will see later (Corollary (2.6)).

There is a similar homomorphism $R: \widetilde{\operatorname{Diff}}_{\Omega}\left(D^{2}\right) \longrightarrow \mathbf{R}$, where $\widetilde{\operatorname{Diff}}_{\Omega}\left(D^{2}\right)$ denotes the universal covering group of $\operatorname{Diff}_{\Omega}\left(D^{2}\right)$.

Let $\mathcal{L}_{\Omega}\left(D^{2}\right)$ denote the Lie algebra of the divergence free vector fields on the disk which are tangent to the boundary.

Lemma (1.2). For an element $X$ of $\mathcal{L}_{\Omega}\left(D^{2}\right)$, there is a unique function $f_{X}$ on $D^{2}$ such that $d f_{X}=i(X) \Omega$ and $f_{X}$ is zero on $\partial D^{2}$.

Proof. The existence of a function $f_{X}$ such that $d f_{X}=i(X) \Omega$ is classical. Since $X$ is tangent to the boundary, i.e., tangent to the rotational vector field $Z$, for the function $f_{X}$, we have

$$
Z f_{X}=i(Z) d f_{X}=i(Z) i(X) \Omega=0 .
$$

Thus $f_{X}$ is constant along the boundary. So we can choose the function $f_{X}$ which is zero along the boundary. 
Lemma (1.3) ([1, Lemme II.4.2]). For $X \in \mathcal{L}_{\Omega}\left(D^{2}\right)$, let $f_{X}$ denote the function on $D^{2}$ such that $d f_{X}=i(X) \Omega$ and $f_{X}$ is zero on $\partial D^{2}$. Let $\mathcal{R}: \mathcal{L}_{\Omega}\left(D^{2}\right) \longrightarrow \mathbf{R}$ be the map defined by

$$
\mathcal{R}(X)=\int_{D^{2}} f_{X} \Omega
$$

Then $\mathcal{R}$ is a surjective Lie algebra homomorphism.

In fact, note here the useful formulae ([1, Lemme II.4.2]), for $X, Y \in \mathcal{L}_{\Omega}\left(D^{2}\right)$,

$$
f_{[X, Y]}=i(X) i(Y) \Omega \quad \text { and } \quad f_{[X, Y]} \Omega=d f_{Y} \wedge d f_{X} .
$$

Thus

$$
\mathcal{R}([X, Y])=\int_{D^{2}} d f_{Y} \wedge d f_{X}=\int_{D^{2}} d\left(f_{Y} \wedge d f_{X}\right)=\int_{\partial D^{2}} f_{Y} d f_{X}=0 .
$$

Note also that for the rotational vector field $Z, i(Z) \Omega=-r d r=d\left(\left(1-r^{2}\right) / 2\right)$ and

$$
\mathcal{R}(Z)=\int_{D^{2}} \frac{1}{2}\left(1-r^{2}\right) r d r d \theta=\pi\left[\frac{r^{2}}{2}-\frac{r^{4}}{4}\right]_{0}^{1}=\frac{\pi}{4} .
$$

Now we can define $R: \widetilde{\operatorname{Diff}}_{\Omega}\left(D^{2}\right) \longrightarrow \mathbf{R}$ as in [1, Lemme II.4.1]. Let $h_{t} \in$ $\operatorname{Diff}_{\Omega}\left(D^{2}\right)(t \in[0,1])$ be a smooth path from the identity. Let $X_{t}$ be the vector field defined by $X_{t}(x)=\left(\partial h_{t} / \partial t\right)\left(h_{t}^{-1}(x)\right)$. Then $X_{t}$ is an element of $\mathcal{L}_{\Omega}\left(D^{2}\right)$. For the element $\left[h_{t}\right] \in \widetilde{\operatorname{Diff}}_{\Omega}\left(D^{2}\right)$, put

$$
R\left(\left[h_{t}\right]\right)=\int_{0}^{1} \mathcal{R}\left(X_{t}\right) d t
$$

The verification of the well definedness of $R$ is the same as in [1, Lemme II.4.1].

Lemma (1.4) ([1, Lemme II.4.1]). $R: \widetilde{\operatorname{Diff}}_{\Omega}\left(D^{2}\right) \longrightarrow \mathbf{R}$ is a well-defined homomorphism.

Note that for a full rotation $T$,

$$
R(T)=\int_{0}^{1} \mathcal{R}(2 \pi Z) d t=\frac{\pi^{2}}{2} .
$$

The following lemma shows that $\rho$ and $R$ restricted on $\operatorname{Diff}_{\Omega}\left(D^{2}, \partial D^{2}\right)$ are essentially the same. Note that we will show later that $\operatorname{Diff}_{\Omega}\left(D^{2}, \partial D^{2}\right)$ is contractible (Corollary (2.6)).

Lemma (1.5) ([1, Proposition II.4.3]). Let $h_{t}$ be a path in $\operatorname{Diff}_{\Omega}\left(D^{2}, \partial D^{2}\right)$ such that $h_{0}=\mathrm{id}$, then $\rho\left(h_{1}\right)=2 R\left(\left[h_{t}\right]\right)$.

In fact, for $X_{t}(x)=\left(\partial h_{t} / \partial t\right)\left(h_{t}^{-1}(x)\right)$, the function $f_{X_{t}}$ satisfies the following identity ([1, Proposition II.4.3]).

$$
h_{1}^{*} \lambda-\lambda=d\left(\int_{0}^{1} h_{t}^{*} f_{X_{t}} d t+\int_{0}^{1} h_{t}^{*} i\left(X_{t}\right) \lambda d t\right) .
$$

Note also the following identity ([1, Proposition II.4.3]).

$$
\left(i\left(X_{t}\right) \lambda\right) \Omega=\lambda \wedge d f_{X_{t}}=-d\left(f_{X_{t}} \lambda\right)+f_{X_{t}} \Omega .
$$


Thus

$$
\begin{aligned}
\rho\left(h_{1}\right) & =\int_{D^{2}}\left(\int_{0}^{1} h_{t}^{*} f_{X_{t}} d t+\int_{0}^{1} h_{t}^{*} i\left(X_{t}\right) \lambda d t\right) \Omega \\
& =\int_{0}^{1}\left[\int_{D^{2}} f_{X_{t}} \Omega\right] d t+\int_{0}^{1}\left[\int_{D^{2}}\left(i\left(X_{t}\right) \lambda\right) \Omega\right] d t \\
& =2 \int_{0}^{1} \int_{D^{2}} f_{X_{t}} \Omega d t+\int_{0}^{1} \int_{\partial D^{2}} f_{X_{t}} \lambda d t \\
& =2 R\left(\left[h_{t}\right]\right) .
\end{aligned}
$$

\section{Surjectivity of $\operatorname{Diff} \Omega(M)_{0} \rightarrow \operatorname{Diff}(\partial M)_{0}$}

We review here the relationship between the group of volume preserving diffeomorphisms of a compact manifold with boundary and the group of diffeomorphisms of the boundary, which is well known to the experts.

Let $M$ be a compact oriented manifold with boundary $\partial M$. Let $\operatorname{Diff}(M)$ denote the group of orientation preserving $C^{\infty}$ diffeomorphisms of $M$ with the $C^{\infty}$ topology. Let $\operatorname{Diff}_{\Omega}(M)$ denote the group of $C^{\infty}$ diffeomorphisms of $M$ which preserve the volume form $\Omega$. By a result of Moser ([12]), the homomorphism $\operatorname{Diff}_{\Omega}(M) \longrightarrow \operatorname{Diff}(M)$ induces a bijection on $\pi_{0}$.

We look at the homomorphism $\operatorname{Diff}_{\Omega}(M)_{0} \longrightarrow \operatorname{Diff}(\partial M)_{0}$ between their identity components.

Let $\mathcal{L}_{\Omega}(M)$ denote the Lie algebra of the divergence free vector fields on $M$ tangent to the boundary $\partial M$. The following lemma is well known.

Lemma (2.1). For an element $X$ of $\mathcal{L}_{\Omega}(M)$, there is a $(\operatorname{dim}(M)-2)$-form $\alpha_{X}$ on a neighborhood of $\partial M$ such that $d \alpha_{X}=i(X) \Omega$. For a continuous family of elements $X_{(w)}(w \in W)$ of $\mathcal{L}_{\Omega}(M)$, a family of $(\operatorname{dim}(M)-2)$-forms $\alpha_{X_{(w)}}$ on a neighborhood of $\partial M$ can be taken continuously on $w$.

Lemma (2.2) ([8, Theorem 1]). The homomorphism $\operatorname{Diff}_{\Omega}(M)_{0} \longrightarrow \operatorname{Diff}(\partial M)_{0}$ is surjective.

Proof. For a vector field $\xi$ on $\partial M$, we show that there is a divergence free vector field on $M$ tangent to the boundary $\partial M$ which is an extension of $\xi$. In fact, in a collar neighborhood $\partial M \times[0,1]$ of $\partial M=\partial M \times\{0\}$, by a theorem of Moser ([12]), we may assume that the volume form $\Omega_{M}$ is of the form $\Omega_{\partial M} \wedge d s$. We $\operatorname{define} \operatorname{div}(\xi)$ by $\operatorname{div}(\xi) \Omega_{\partial M}=L_{\xi} \Omega_{\partial M}$. We put $X(y, t)=\xi(y)-s \operatorname{div}(\xi)(y)(\partial / \partial s)$. Then on the collar neighborhood $\partial M \times[0,1]$,

$$
\begin{aligned}
L_{X(y, t)} \Omega_{M} & =L_{\xi(y)}\left(\Omega_{\partial M} \wedge d s\right)-L_{s \operatorname{div}(\xi)(y)(\partial / \partial s)}\left(\Omega_{\partial M} \wedge d s\right) \\
& =\operatorname{div}(\xi) \Omega_{\partial M} \wedge d s-(-1)^{\operatorname{dim} M-1} d\left(s \operatorname{div}(\xi)(y) \Omega_{\partial M}\right)=0 .
\end{aligned}
$$

Then in a neighborhood of $\partial M$, there is a $(\operatorname{dim}(M)-2)$-form $\alpha_{X}$ such that $d \alpha_{X}=$ $i(X) \Omega_{M}$. Now we choose a smooth function $\mu$ on $\partial M \times[0,1]$ which is 1 in a neighborhood of $\partial M=\partial M \times\{0\}$ and is 0 on a neighborhood of $\partial M \times\{1\}$. Then the vector field $Y$ which satisfies $d\left(\mu \alpha_{X}\right)=i(Y) \Omega_{M}$ is the desired extension. Thus we proved Lemma (2.2).

In the case where $M=D^{2}$, we have a little more explicit expression. For a vector field $\xi=\xi(\partial / \partial \theta)$ on $\partial D^{2}$, define a function $f$ on $D^{2}-\{O\}$ by $f(r, \theta)=$ $\left(1-r^{2} / 2\right) \xi(\theta)$. Let $\mu$ be a smooth function on $D^{2}$ which is 1 in a neighborhood 
of $\partial D^{2}$ and is 0 on a neighborhood of $O$. Then the vector field $Y$ defined by $d(\mu f)=i(Y) \Omega$ is the desired extension.

Since we have shown that $\operatorname{Diff}_{\Omega}(M)_{0} \longrightarrow \operatorname{Diff}(\partial M)_{0}$ is surjective, we are interested in the kernel of the homomorphism. Let $\widetilde{\operatorname{Diff}}_{\Omega}(M, \partial M)_{0}, \widetilde{\operatorname{Diff}}_{\Omega}(M)_{0}$ and $\widetilde{\operatorname{Diff}}(\partial M)_{0}$ be the universal covering groups of $\operatorname{Diff}_{\Omega}(M, \partial M)_{0}, \operatorname{Diff}_{\Omega}(M)_{0}$ and $\operatorname{Diff}(\partial M)_{0}$, respectively. We have the following corollary.

Corollary (2.3). The kernel of the surjective homomorphism

$$
\widetilde{\operatorname{Diff}}_{\Omega}(M)_{0} \longrightarrow \widetilde{\operatorname{Diff}}(\partial M)_{0}
$$

coincides with $\widetilde{\operatorname{Diff}}_{\Omega}(M, \partial M)_{0}$.

By a result of Moser ([12]), the homomorphism $\operatorname{Diff}_{\Omega}(M, \partial M) \longrightarrow \operatorname{Diff}(M, \partial M)$ induces a bijection on $\pi_{0}$. The following proposition gives the information on the topology of the identity component $\operatorname{Diff} \Omega(M, \partial M)_{0}$.

Proposition (2.4). Let $M$ be a connected oriented manifold with non-empty boundary $\partial M$. The homotopy fiber of $\operatorname{Diff}_{\Omega}(M, \partial M)_{0} \longrightarrow \operatorname{Diff}(M, \partial M)_{0}$ is contractible.

Proof. Let $h: S^{q-1} \longrightarrow \operatorname{Diff}_{\Omega}(M, \partial M)$ be a smooth map. We assume that we have a smooth extension $H: D^{q} \longrightarrow \operatorname{Diff}(M, \partial M)$ of $h$. We construct Moser's homotopy $([12])$. Let $\Omega_{t}^{(w)}=(1-t) H(w)^{*} \Omega+t \Omega$. Since $M$ is connected and with non-empty boundary, there exists a $(\operatorname{dim}(M)-1)$-form $\lambda$ such that $d \lambda=\Omega$. Note that

$$
\int_{M} H(w)^{*} \Omega=\int_{\partial M}(H(w) \mid \partial M)^{*} \lambda=\int_{\partial M} \lambda=\int_{M} \Omega .
$$

Put $\alpha_{w}=H(w)^{*} \lambda-\lambda$. Then $d \alpha_{w}=H(w)^{*} \Omega-\Omega$. Since $\alpha_{w} \mid \partial M=0$, on $M \times[0,1]$ we can write

$$
\alpha_{w}=u_{w}(y, t) \wedge d t+v_{w}(y, t) \Omega_{\partial M},
$$

where $\Omega_{\partial M}$ is the volume form of $\partial M$ and $v(y, 0)=0$. Let $\beta_{w}=\alpha_{w}-d\left(\mu \cdot u_{w}(y, 0) t\right)$, where $\mu$ is a function of $M$ which is 1 in a neighborhood of $\partial M=M \times\{0\}$ and is 0 in a neighborhood of $\partial M=M \times\{1\}$. Then $d \beta_{w}=H(w)^{*} \Omega-\Omega$ and $\beta_{w}(y)=0$ for $y \in \partial M$. Let $X_{t}^{(w)}$ be the vector field such that $i\left(X_{t}^{(w)}\right) \Omega_{t}^{(w)}=-\beta_{w}$. Let $\varphi_{t}^{(w)}$ be the diffeomorphism of $M$ such that $\left(\partial \varphi_{t}^{(w)} / \partial t\right)\left(\varphi_{t}^{(w)}(x)\right)=X_{t}^{(w)}\left(\varphi_{t}^{(w)}(x)\right)$. Then

$$
\begin{aligned}
(\partial / \partial t)\left(\varphi_{t}^{(w)}\right)^{*} \Omega_{t}^{(w)} & =\left(\varphi_{t}^{(w)}\right)^{*}\left(L_{X_{t}^{(w)}} \Omega_{t}^{(w)}+(\partial / \partial t) \Omega_{t}^{(w)}\right) \\
& =\left(\varphi_{t}^{(w)}\right)^{*}\left(d i\left(X_{t}^{(w)}\right) \Omega_{t}^{(w)}+H(w)^{*} \Omega-\Omega\right)=0 .
\end{aligned}
$$

Thus $\left(\varphi_{1}^{(w)}\right)^{*} \Omega=H(w)^{*} \Omega$ for $w \in D^{q}$, and $\left(\varphi_{t}^{(w)}\right)^{*} \Omega=\Omega$ for $w \in \partial D^{q}$. Put

$$
H^{\prime}(w)=\left\{\begin{aligned}
H(w /\|w\|) \circ\left(\varphi_{2(1-\|w\|)}^{(w /\|w\|)}\right)^{-1} & \text { for } \quad\|w\| \geq 1 / 2 \quad \text { and } \\
H(2 w)\left(\varphi_{1}^{(2 w)}\right)^{-1} & \text { for } \quad\|w\| \leq 1 / 2 .
\end{aligned}\right.
$$

Then $H^{\prime}(w) \in \operatorname{Diff}_{\Omega}(M, \partial M)_{0}$.

We remark here that, as A. Marin pointed out to me, Proposition (2.4) is true even if $\partial M=\emptyset$. This can be shown by obtaining $\alpha_{w}$ in the proof of Proposition (2.4), using the tensor product of topological vector spaces as in the proof of $[1$, Lemme II.2.2]. 
Proposition (2.5). Let $M$ be a manifold without boundary. The homotopy fiber of $\operatorname{Diff}_{\Omega}(M)_{0} \longrightarrow \operatorname{Diff}(M)_{0}$ is contractible.

Now we return to the group of area preserving diffeomorphism of the disk. By a result of Smale ([14]), Diff $\left(D^{2}, \partial D^{2}\right)$ is contractible. Hence we obtain the following corollary.

Corollary (2.6). $\operatorname{Diff}_{\Omega}\left(D^{2}, \partial D^{2}\right)$ is contractible.

By this corollary, Diff ${ }_{\Omega}\left(D^{2}, \partial D^{2}\right)=\widetilde{\operatorname{Diff}}_{\Omega}\left(D^{2}, \partial D^{2}\right)$ coincides with the kernel of $\operatorname{Diff} \Omega\left(D^{2}\right) \longrightarrow \operatorname{Diff}\left(S^{1}\right)_{0}$, and we have the following exact sequence of contractible topological groups

$$
1 \longrightarrow \operatorname{Diff}_{\Omega}\left(D^{2}, \partial D^{2}\right) \longrightarrow \widetilde{\operatorname{Diff}_{\Omega}}\left(D^{2}\right)_{0} \longrightarrow \widetilde{\operatorname{Diff}}\left(S^{1}\right)_{0} \longrightarrow 1
$$

Note that $\operatorname{Diff}\left(S^{1}\right)_{0} \simeq S^{1}$.

Now we can make some computation.

First note the following corollary.

Corollary (2.7). Let $h$ be an element of $\operatorname{Diff}_{\Omega}\left(D^{2}, \partial D^{2}\right)$. For any path $h_{t}$ in $\operatorname{Diff}_{\Omega}\left(D^{2}, \partial D^{2}\right)$ such that $h_{0}=\mathrm{id}$ and $h_{1}=h, \rho(h)=2 R\left(\left[h_{t}\right]\right)$.

In the following corollaries, the path $h_{t} \mid \partial D^{2}$ in $\operatorname{Diff}\left(S^{1}\right)_{0}$ may not be a trivial path.

Corollary (2.8). Let $h_{t}$ be a path in $\operatorname{Diff}_{\Omega}\left(D^{2}\right)$ such that $h_{0}=\mathrm{id}$ and $\left[h_{t} \mid \partial D^{2}\right]=$ id $\in \widetilde{\operatorname{Diff}}\left(S^{1}\right)_{0}$. Then $\rho\left(h_{1}\right)=2 R\left(\left[h_{t}\right]\right)$.

Proof. Let $h_{t}^{\prime}$ be a path in $\operatorname{Diff}_{\Omega}\left(D^{2}, \partial D^{2}\right)$ such that $h_{0}^{\prime}=$ id and $h_{1}^{\prime}=h_{1}$. Since $h_{t}^{\prime} \mid \partial D^{2}$ is a trivial path in $\operatorname{Diff}\left(S^{1}\right)$ and is homotopic to $h_{t} \mid \partial D^{2}$, we have $\left[h_{t}^{\prime}\right]=\left[h_{t}\right]$ in $\widetilde{\operatorname{Diff}} \Omega\left(D^{2}\right)$ and $R\left(\left[h_{t}^{\prime}\right]\right)=R\left(\left[h_{t}\right]\right)$. On the other hand, $\rho\left(h_{1}\right)=\rho\left(h_{1}^{\prime}\right)=2 R\left(\left[h_{t}^{\prime}\right]\right)$. Thus $\rho\left(h_{1}\right)=2 R\left(\left[h_{t}\right]\right)$.

Corollary (2.9). Let $h_{t}$ be a path in $\operatorname{Diff}_{\Omega}\left(D^{2}\right)$ such that $h_{0}=\mathrm{id}$ and $\left[h_{t} \mid \partial D^{2}\right]=$ $T_{k} \in \widetilde{\operatorname{Diff}}\left(S^{1}\right)_{0}$, where $T_{k}$ is the translation by $k \in \mathbf{Z}$. Then $\rho\left(h_{1}\right)=2 R\left(\left[h_{t}\right]\right)-k \pi^{2}$.

Proof. Let $h_{t}^{\prime \prime}$ be the path $h_{t}$ followed by $-k$ times the full rotation. Then by the precedent corollary,

$$
\rho\left(h_{1}\right)=\rho\left(h_{1}^{\prime \prime}\right)=2 R\left(\left[h_{t}^{\prime \prime}\right]\right)=2\left(R\left(\left[h_{t}\right]\right)-k\left(\pi^{2} / 2\right)\right) .
$$

\section{EULER CLASS}

Let Diff $\left(S^{1}\right)_{0}$ denote the group of $C^{\infty}$ diffeomorphisms of the circle isotopic to the identity with the $C^{\infty}$ topology. Let $\widetilde{\operatorname{Diff}}\left(S^{1}\right)_{0}$ denote the universal covering of $\operatorname{Diff}\left(S^{1}\right)_{0}$. $\widetilde{\operatorname{Diff}}\left(S^{1}\right)_{0}$ is the group of the periodic diffeomorphisms of the real line and there is a homomorphism $\widetilde{\operatorname{Diff}}\left(S^{1}\right)_{0} \longrightarrow \operatorname{Diff}\left(S^{1}\right)_{0}$ whose kernel is $\mathbf{Z}$.

Let $\Sigma_{\mathbf{g}}$ denote the closed oriented surface of genus $\mathbf{g}$. Its fundamental group has the well known presentation

$$
\left\langle a_{1}, \cdots, a_{2 \mathbf{g}} ;\left[a_{1}, a_{2}\right] \cdots\left[a_{2 \mathbf{g}-1}, a_{2 \mathbf{g}}\right]\right\rangle .
$$

For a homomorphism $\psi: \pi_{1}\left(\Sigma_{\mathbf{g}}\right) \longrightarrow \operatorname{Diff}\left(S^{1}\right)_{0}$, we take the lifts $\widetilde{g}_{i}$ to $\widetilde{\operatorname{Diff}}\left(S^{1}\right)_{0}$ for each $g_{i}=\psi\left(a_{i}\right)$. Then $\left[\widetilde{g_{1}}, \widetilde{g_{2}}\right] \cdots\left[\widetilde{g_{2 \mathrm{~g}-1}}, \widetilde{g_{2 \mathrm{~g}}}\right]$ is a translation by an integer. This integer is equal to $e(\psi)\left[\Sigma_{\mathbf{g}}\right]$, the Euler class of the associated circle bundle evaluated on the fundamental class of the surface (see Milnor [10] and Wood [15]). 
The Euler class is also written as the cocycle defined by using a section $s$ : $\operatorname{Diff}\left(S^{1}\right)_{0} \longrightarrow \widetilde{\operatorname{Diff}}\left(S^{1}\right)_{0}$. Now for elements $g_{1}, g_{2}$ of $\operatorname{Diff}\left(S^{1}\right)_{0}$, put

$$
e\left(g_{1}, g_{2}\right)=s\left(g_{1}\right) \circ s\left(g_{2}\right) \circ\left(s\left(g_{1} \circ g_{2}\right)\right)^{-1} \in \mathbf{Z} \text {. }
$$

Then the fact that $\mathbf{Z}$ is the center of $\widetilde{\operatorname{Diff}}\left(S^{1}\right)_{0}$ implies that $e\left(g_{1}, g_{2}\right)$ is a 2-cocycle of the group $\operatorname{Diff}\left(S^{1}\right)_{0}$.

We are going to show that by choosing a section $\sigma: \widetilde{\operatorname{Diff}}\left(S^{1}\right)_{0} \longrightarrow \widetilde{\operatorname{Diff}_{\Omega}}\left(D^{2}\right)$, we can define a 2-cocycle of $\operatorname{Diff}\left(S^{1}\right)_{0}$ which is cohomologous to $-\pi^{2} e$. Let $p$ : $\widetilde{\operatorname{Diff}}_{\Omega}\left(D^{2}\right) \longrightarrow \operatorname{Diff}_{\Omega}\left(D^{2}\right)$ denote the projection.

For elements $g_{1}, g_{2}$ of $\operatorname{Diff}\left(S^{1}\right)_{0}$, put

$$
c\left(g_{1}, g_{2}\right)=\rho\left(p\left(\sigma s\left(g_{1}\right) \circ \sigma s\left(g_{2}\right) \circ\left(\sigma s\left(g_{1} \circ g_{2}\right)\right)^{-1}\right)\right) \in \mathbf{R} .
$$

Note that $p\left(\sigma s\left(g_{1}\right) \circ \sigma s\left(g_{2}\right) \circ\left(\sigma s\left(g_{1} \circ g_{2}\right)\right)^{-1}\right)=p\left(\sigma s\left(g_{1}\right)\right) \circ p\left(\sigma s\left(g_{2}\right)\right) \circ p\left(\sigma s\left(g_{1} \circ g_{2}\right)\right)^{-1}$ is an element of $\operatorname{Diff}_{\Omega}\left(D^{2}, \partial D^{2}\right)$. Now by Corollary $(2.9)$, we see the following:

$$
\begin{aligned}
& \rho\left(p\left(\sigma s\left(g_{1}\right) \circ \sigma s\left(g_{2}\right) \circ\left(\sigma s\left(g_{1} \circ g_{2}\right)\right)^{-1}\right)\right) \\
& \quad=2 R\left(\sigma s\left(g_{1}\right) \circ \sigma s\left(g_{2}\right) \circ\left(\sigma s\left(g_{1} \circ g_{2}\right)\right)^{-1}\right)-\pi^{2}\left(s\left(g_{1}\right) \circ s\left(g_{2}\right) \circ\left(s\left(g_{1} \circ g_{2}\right)\right)^{-1}\right) \\
& \quad=2 R\left(\sigma s\left(g_{1}\right)\right)+2 R\left(\sigma s\left(g_{2}\right)\right)-2 R\left(\sigma s\left(g_{1} \circ g_{2}\right)\right)-\pi^{2}\left(s\left(g_{1}\right) \circ s\left(g_{2}\right) \circ\left(s\left(g_{1} \circ g_{2}\right)\right)^{-1}\right) .
\end{aligned}
$$

Put $\delta(f)=2 R(\sigma s(f))$. Then we have shown the following proposition.

Proposition (3.1). $c\left(g_{1}, g_{2}\right)=\delta\left(\partial\left(g_{1}, g_{2}\right)\right)-\pi^{2} e\left(g_{1}, g_{2}\right)$.

This shows that $c\left(g_{1}, g_{2}\right)$ and $-\pi^{2} e\left(g_{1}, g_{2}\right)$ are cohomologous.

Now we prove Theorem A.

Proof of Theorem A. For a homomorphism $\psi: \pi_{1}\left(\Sigma_{\mathbf{g}}\right) \longrightarrow \operatorname{Diff}\left(S^{1}\right)_{0}$, we have a triangulation of $\Sigma_{\mathbf{g}}$ with one vertex such that the sum of the triangles gives the fundamental class. We assume that the edges contain the generators $a_{1}, \cdots, a_{2}$ of $\pi_{1}\left(\Sigma_{\mathbf{g}}\right)$.

For each edge of the triangle, we have a diffeomorphism $g$ of the circle which is the holonomy along this edge of the foliated $S^{1}$ bundle, and the extension $h \in \operatorname{Diff}_{\Omega}\left(D^{2}\right)$ (arbitrarily chosen for edges other than $a_{1}, \cdots, a_{2 \mathbf{g}}$ ). Hence for a triangle with holonomies $g_{1}, g_{2}$ and $g_{1} \circ g_{2}$, we have the real number $\rho\left(h_{1} \circ h_{2} \circ\left(h_{12}\right)^{-1}\right)$. The sum of them over the triangles gives $\rho\left(\left[h_{1}, h_{2}\right] \cdots\left[h_{2 \mathbf{g}-1}, h_{2 \mathbf{g}}\right]\right)$. We show that the sum does not depend on the choice of the extension. Then since the choice $p(\sigma s(g))$ gives the Euler class, another choice also gives it.

For a triangle with holonomies $g_{1}, g_{2}$ and $g_{1} \circ g_{2}$, let $h_{1}, h_{2}$ and $h_{12}$, and $h_{1}^{\prime}, h_{2}^{\prime}$ and $h_{12}^{\prime}$ be the extensions of them. Then

$$
\begin{aligned}
& \rho\left(h_{1}^{\prime} \circ h_{2}^{\prime} \circ\left(h_{12}^{\prime}\right)^{-1}\right) \\
= & \rho\left(h_{1}^{\prime} \circ\left(h_{1}^{-1} \circ h_{1}\right) \circ h_{2}^{\prime} \circ\left(h_{2}^{-1} \circ h_{1}^{-1} \circ h_{1} \circ h_{2}\right) \circ\left(\left(h_{12}\right)^{-1} \circ h_{12}\right) \circ\left(h_{12}^{\prime}\right)^{-1}\right) \\
= & \rho\left(\left(h_{1}^{\prime} \circ h_{1}^{-1}\right) \circ\left[h_{1} \circ\left(h_{2}^{\prime} \circ h_{2}^{-1}\right) \circ h_{1}^{-1}\right]\right. \\
& \left.\circ\left(h_{1} \circ h_{2} \circ\left(h_{12}\right)^{-1}\right) \circ\left(h_{12}^{\prime} \circ\left(h_{12}\right)^{-1}\right)^{-1}\right) \\
= & \rho\left(h_{1}^{\prime} \circ h_{1}^{-1}\right)+\rho\left(h_{1} \circ\left(h_{2}^{\prime} \circ h_{2}^{-1}\right) \circ h_{1}^{-1}\right) \\
& \quad+\rho\left(h_{1} \circ h_{2} \circ\left(h_{12}\right)^{-1}\right)+\rho\left(\left(h_{12}^{\prime} \circ\left(h_{12}\right)^{-1}\right)^{-1}\right) \\
= & \rho\left(h_{1}^{\prime} \circ h_{1}^{-1}\right)+\rho\left(h_{2}^{\prime} \circ h_{2}^{-1}\right)+\rho\left(h_{1} \circ h_{2} \circ\left(h_{12}\right)^{-1}\right)-\rho\left(h_{12}^{\prime} \circ\left(h_{12}\right)^{-1}\right) .
\end{aligned}
$$

This shows that the sum of $\rho\left(h_{1}^{\prime} \circ h_{2}^{\prime} \circ\left(h_{12}^{\prime}\right)^{-1}\right)$ over the triangles are the same as the sum of $\rho\left(h_{1} \circ h_{2} \circ\left(h_{12}\right)^{-1}\right)$ over the triangles. 
Corollary (3.2). Let $\Sigma_{g}$ be a closed surface of genus $g$. If a homomorphism $\pi_{1}\left(\Sigma_{\mathbf{g}}\right) \longrightarrow \operatorname{Diff}\left(S^{1}\right)_{0}$ extends as a homomorphism $\pi_{1}\left(\Sigma_{\mathbf{g}}\right) \longrightarrow \operatorname{Diff}_{\Omega}\left(D^{2}\right)$, then the associated $S^{1}$ or $D^{2}$ bundle is trivial.

This fact has been known by a much stronger result of Mitsumatsu ([11], [7]) which says that if a foliated $D^{n}$ bundle over an $n$ dimensional manifold admits a transversely invariant measure whose support consists of more than one point transversely, then its Euler class is zero.

In our case, this fact for a flat $\operatorname{Diff} \Omega_{\Omega}\left(D^{n}\right)_{0}$ bundle can also be shown as follows. Consider the Thom class of the disk bundle $E \longrightarrow M$. Since the Thom class is characterized by the fact that the restriction to the fiber $\left(D^{n}, \partial D^{n}\right)$ is the generator of $H^{n}\left(D^{n}, \partial D^{n}\right)$. When there is a transverse invariant volume form, then we can give a Thom form $\Phi$ which is locally the volume form of the fiber in each foliated chart. Then it is clear that $\Phi \wedge \Phi=0$. Since the Euler class $e$ satisfies $[\Phi \wedge \Phi]=$ $[\Phi] \cup \operatorname{proj}^{*} e$, and $[\Phi] \cup: H^{*}(M) \longrightarrow H^{*+n}(E, \partial E)$ is isomorphic, $\operatorname{proj}^{*} e=0$, hence $e=s^{*}$ proj $^{*} e=0$. Thus the Euler class $e$ is zero.

In view of this corollary, it is interesting to investigate the expression of the Euler class for the foliated sphere bundle in terms of the volume preserving extensions of its holonomy. In other words, it is interesting to look for a result similar to our main theorem for some cohomology classes of groups $\operatorname{Diff}\left(S^{q-1}\right)_{0}$ and $\operatorname{Diff} \Omega_{\Omega}\left(D^{q}\right)_{0}$.

The Euler class is defined for topological sphere bundles. In our case it is defined as a 2-dimensional cohomology class of the group $\operatorname{Homeo}\left(S^{1}\right)_{0}$. Hence it is interesting to know about homology groups of the group Homeo $\Omega\left(D^{2}\right)_{0}$ of measure preserving homeomorphisms. For the group of homeomorphisms of a compact manifold of dimension greater than 2 which preserve the Lebesgue measure, by using a result of Oxtoby and Ulam ([13]), Fathi calculated its abelianization ([3]). The abelianization of $\operatorname{Homeo}_{\Omega}\left(D^{2}\right)_{0}$ or $\operatorname{Homeo}_{\Omega}\left(D^{2}, \partial D^{2}\right)$ is not known.

\section{REFERENCES}

1. A. Banyaga, Sur la structure du groupe des difféomorphismes qui préservent une forme symplectique, Comment. Math. Helvetici 53 (1978), 174-227. MR 80c:58005

2. E. Calabi, On the group of automorphisms of a symplectic manifold, Problems in Analysis (symposium in honour of S. Bochner), Princeton Univ. Press (1970), 1-26. MR 50:3268

3. A. Fathi, Structure of the group of homeomorphisms preserving a good measure on a compact manifold, Ann. Sci. Ecole Norm. Sup. (4) 13 (1980), 45-93. MR 81k:58042

4. J.-M. Gambaudo and E. Ghys, Enlacement asymptotiques, Topology 36 (1997), 1355-1379. MR 98f: 57050

5. P. Greenberg, Generators and relations in the classifying space for pl foliations, Topology Appl. 48 (1992), 185-205. MR 94c:57047

6. H. Hofer and E. Zehnder, Symplectic invariants and Hamiltonian dynamics, Birkhäuser Verlag (1994). MR 96g:58001

7. S. Hurder and Y. Mitsumatsu, Transverse Euler class of foliations on nonatomic foliation cycles, Contemp. Math. 161 (1994), 29-39. MR 95e:57049

8. A. B. Krygin, Continuation of diffeomorphisms retaining volume, Funct. Anal. and Appl. 5 (1971), 147-150.

9. D. McDuff, Local homology of groups of volume preserving diffeomorphisms, Ann. Sci. Ecole Norm. Sup. (4) 15 (1982), 609-648. MR 85i:58028

10. J. Milnor, On the existence of a connection with curvature zero, Comment. Math. Helv. 32 (1958), 215-233. MR 20:2020

11. Y. Mitsumatsu, On the self-intersections of foliation cycles, Trans. Amer. Math. Soc. 334 (1992), 851-860. MR 94c:57049

12. J. Moser, On the volume elements on a manifold, Trans. Amer. Math. Soc. 120 (1965), 286-294. MR 32:409 
13. J. C. Oxtoby and S. M. Ulam, Measure preserving homeomorphisms and metrical transitivity, Annals of Math. 42 (1941), 874-920. MR 3:211b

14. S. Smale, Diffeomorphisms of the 2-sphere, Proc. Amer. Math. Soc. 10 (1959), 621-626. MR 22:3004

15. J. Wood, Bundles with totally disconnected structure groups, Comment. Math. Helv. 46 (1971), 257-273. MR 45:2732

Graduate School of Mathematical Sciences, University of Tokyo, Komaba Meguro, TOKYO 153, JAPAN

E-mail address: tsuboi@ms.u-tokyo.ac.jp 